\title{
Comparison of Terrestrial DTV Systems: ISDB-TB and ATSC 3.0
}

\author{
Victor M. Dionísio \\ Cristiano Akamine
}




\title{
Comparison of Terrestrial DTV Systems: ISDB- $\mathrm{T}_{\mathrm{B}}$ and ATSC 3.0
}

\author{
Victor M. Dionísio and Cristiano Akamine \\ Electrical Engineering and Computing Program \\ Mackenzie Presbyterian University \\ Sao Paulo, Brazil \\ victor.dionisio@ieee.org, akamine@ieee.org
}

\begin{abstract}
This review discusses the ISDB-T T $_{B}$ and ATSC 3.0 systems, comparing the differences between the Input Formatting, passing by video compression, data multiplexing and encapsulation, the Channel coding, Interleavers, Constellation Mappers, Multiplexing methods, Framing, Waveform generation and synchronism. Finally, a performance comparison, using similar parameters, between bitrate and required Carrier-to-Noise ratio is presented.
\end{abstract}

Index Terms-Digital Terrestrial Television Broadcasting, Making Better use of spectrum, Next generation of broadcasting systems, ISDB-T, ATSC 3.0.

\section{INTRODUCTION}

The Integrated Services Digital Broadcasting - Terrestrial version B (ISDB-T $\mathrm{B}$ ) system was officially launched in 2007 , in Brazil. Throughout this time, the innovative technologies for Communications systems and new services that were developed are no longer compatible with the ISDB-T $\mathrm{B}_{\mathrm{B}}$.

These modern technologies were created due to the new scenario the Communications systems are in, mainly provided by the internet, smartphones, image quality - Ultra High Definition (UHD) (4K and $8 \mathrm{~K}$ ) - and consequently increase of generated data. Processing this data so it can be transmitted within the limited bandwidth that broadcasters have is the goal of these technologies since part of the spectrum is being reclaimed for broadband wireless services by spectrum authorities [1].

The ISDB- $\mathrm{T}_{\mathrm{B}}$ uses Reed Solomon (RS), Convolutional codes, modulation orders up to 64 constellation points in Quadrature Amplitude Modulation (QAM) and the video compression standard H.264. When compared to recent technologies, the ones used in ISDB- $\mathrm{T}_{\mathrm{B}}$ does not support the scenario mentioned earlier [2].

The need to use the remaining spectrum more efficiently led the Advanced Television Systems Committee (ATSC) and other Standard Development Organizations (SDO) to

This work was supported in part by the Coordination for the Improvement of Higher Education Personnel (CAPES), National Research and Educational Network (RNP), National Counsel of Technological and Scientific Development (CNPq) and MackPesquisa. start researches for new systems using the state-of-the-art technologies. In September 2016, the ATSC released the new system Physical Layer standard, called ATSC 3.0.

The system was adopted by South Korea, which started the transmissions in May 2017 [3].

ATSC 3.0 uses the state-of-the-art technologies to achieve the goal of efficient use of the spectrum such as the LowDensity Parity Check (LDPC) codes along with Bose, RayChaudhuri, and Hocquenghem $(\mathrm{BCH})$ codes, the nonuniform constellations (NUC), and the Layered Division Multiplexing (LDM) that provide a way to transmit different signals, separated by layers, at the same time and frequency [3], [4].

The Physical Layer Pipes (PLP) concept, introduced in the Digital Video Broadcasting - Terrestrial 2 (DVB-T2), is used in ATSC 3.0. Each PLP can be configured independently and it is possible to transmit two PLPs, one in each layer of the LDM.

The system also incorporates the video compression standard H.265, the Dynamic Adaptive Stream over Hypertext Transfer Protocol (DASH) and the MPEG Media Transport (MMT) technologies [3], [4], [5].

This work presents a comparison between ATSC 3.0 and ISDB- $\mathrm{T}_{\mathrm{B}}$. The relevance of this study lies in research ATSC 3.0 so the technologies used in it can serve as a guide to the development of a new broadcast system for Brazil, considering the Analog Switch Off (ASO) that is happening in the country since 2016.

This review is organized in four sections: Section II presents the comparisons between the systems, Section III presents the comparisons between bitrate and required Carrier-to-Noise ratio (CNR), Section IV is the review's conclusion.

\section{SYSTEM COMPARISONS}

\section{A. Input Formatting}

ISDB- $_{B}$ uses the Broadcast Transport Stream (BTS) as the system input. The BTS is created by the re-multiplexing of MPEG-2 TS from the source coding scheme, data carousel etc. so they are in a synchronized order. 
The video is compressed using the H.264 standard, and the audio utilizes the MPEG-4 HE AAC audio standard [2][6].

The ATSC 3.0 system uses a general packet called ATSC Link-layer Protocol (ALP). ALP allows the encapsulation of any packet, such as IP or MPEG-2 TS packets, which means it only processes one type of container. These packets are transported by logic channels called PLPs. Each PLP has its configuration for coding and modulation, and the receiver can recover a maximum of 4 PLPs per frame.

For the video compression, the standard used is the H.265, which has a $50 \%$ improved performance when compared to the H.264. For the audio, the AC-4 audio codec and the MPEG-H audio system are used [3-5], [7-11].

\section{B. Channel Coding}

The codes used in the ISDB-T $\mathrm{T}_{\mathrm{B}}$ system are the Reed Solomon (RS), which is a block code considered a subclass of the Bose Ray-Chaudhuri e Hocquenghem (BCH) code for non-binary symbols, and the Convolutional codes, which make use of memories to code the message.

The RS code used in the system is a shortened version from the $(\mathrm{n}=255, \mathrm{k}=239, \mathrm{t}=8)$, being $(\mathrm{n}=204, \mathrm{k}=188, \mathrm{t}=8)$ since the MPEG-2 TS is standardized at 188 bytes. The generator polynomial $\mathrm{g}(\mathrm{x})_{\mathrm{RS}}$ is given by (1) [6].

$$
\begin{aligned}
& g(x)_{R S}=x^{16}+59 x^{15}+13 x^{14}+104 x^{13}+ \\
& 189 x^{12}+68 x^{11}+209 x^{10}+30 x^{9}+8 x^{8}+ \\
& 163 x^{7}+65 x^{6}+41 x^{5}+229 x^{4}+98 x^{3}+ \\
& 50 x^{2}+36 x^{1}+59
\end{aligned}
$$

For the Convolutional codes, in ISDB-T $_{B}$ they are implemented as a circuit using flip-flops, module 2 adders and multiplexers. Also, a puncturing technique is used to allow a variable code rate. The code rates available are $1 / 2$, $2 / 3,3 / 4,5 / 6$ and $7 / 8$ [1], [6], [12].

In the ATSC 3.0 system, there are three block codes available: Cyclic Redundancy Check (CRC), $\mathrm{BCH}$, both for outer coding, and Low-Density Parity Check (LDPC) for inner coding. Some studies point out that the LDPC code performance can achieve $0.43 \mathrm{~dB}$ of difference from Shannon's theoretical limit [4], [13-15].

There are three possible configurations to use the codes in the system: LDPC only, LDPC concatenated with $\mathrm{BCH}$, which improves the error correction and detection, and LDPC concatenated with $\mathrm{CRC}$, which improves the error detection.

The LDPC codes have two lengths: 16200 and 64800 bits. The 64800 has superior performance but higher latency compared to the 16200 bits. There are 12 different code rates for it: $2 / 15,3 / 15,4 / 15,5 / 15,6 / 15,7 / 15,8 / 15,9 / 15,10 / 15$, $11 / 15,12 / 15$ and $13 / 15$. Also, there are two types of algorithms, A and B, to code the message which is dependent on the code rates. Table I lists the code rates and its algorithms [4], [13-15].

The $\mathrm{BCH}$ codes are used to reduce the LDPC error floor correcting up to 12 bits, therefore improving the error correction as well as error detection capability. It adds 192 parity bits to the message when the LDPC length is 64800 bits and 168 bits when LDPC length is 16200 bits. The generator polynomial $\mathrm{g}(\mathrm{x})_{\mathrm{BCH}}$ is given by $g(x)_{B C H}=$ $g 1(x) g 2(x) \ldots g 12(x)$ and the polynomials for LDPC length 64800 bits are listed in Table II.

CRC codes are only used to improve the error detection capacity of the system. These block codes adds 32 parity bits to the message [4], [13-15].

TABLE I

LDPC CODE RATES FOR ATSC 3.0. ADAPTED FROM [4]

\begin{tabular}{|c|c|c|}
\hline Rate & $\begin{array}{c}\text { Code length } \\
\left(N_{\text {inner }}=16200\right)\end{array}$ & $\begin{array}{c}\text { Code Length } \\
\left(N_{\text {inner }}=64800\right)\end{array}$ \\
\hline \hline $2 / 15$ & $\mathrm{~A}$ & $\mathrm{~A}$ \\
$3 / 15$ & $\mathrm{~A}$ & $\mathrm{~A}$ \\
$4 / 15$ & $\mathrm{~A}$ & $\mathrm{~A}$ \\
$5 / 15$ & $\mathrm{~A}$ & $\mathrm{~A}$ \\
$6 / 15$ & $\mathrm{~B}$ & $\mathrm{~B}$ \\
$7 / 15$ & $\mathrm{~B}$ & $\mathrm{~A}$ \\
$8 / 15$ & $\mathrm{~B}$ & $\mathrm{~B}$ \\
$9 / 15$ & $\mathrm{~B}$ & $\mathrm{~B}$ \\
$10 / 15$ & $\mathrm{~B}$ & $\mathrm{~B}$ \\
$11 / 15$ & $\mathrm{~B}$ & $\mathrm{~B}$ \\
$12 / 15$ & $\mathrm{~B}$ & $\mathrm{~B}$ \\
$13 / 15$ & $\mathrm{~B}$ & $\mathrm{~B}$ \\
\hline
\end{tabular}

TABLE II

BCH POLYNOMIALS. ADAPTED FROM [4]

\begin{tabular}{|c|c|}
\hline$g(x)$ & Code length $\left(N_{\text {inner }}=64800\right)$ \\
\hline \hline$g_{1}(x)$ & $x^{14}+x^{5}+x^{3}+x^{2}+x$ \\
$g_{2}(x)$ & $x^{16}+x^{8}+x^{6}+x^{5}+x^{4}+x+1$ \\
$g_{3}(x)$ & $x^{16}+x^{11}+x^{10}+x^{9}+x^{8}+x^{7}+x^{5}+x^{4}+x^{3}+x^{2}+1$ \\
$g_{4}(x)$ & $x^{16}+x^{14}+x^{12}+x^{11}+x^{9}+x^{6}+x^{4}+x^{2}+1$ \\
$g_{5}(x)$ & $x^{16}+x^{12}+x^{11}+x^{10}+x^{9}+x^{8}+x^{5}+x^{3}+x^{2}+x^{1}+1$ \\
$g_{6}(x)$ & $x^{16}+x^{15}+x^{14}+x^{13}+x^{12}+x^{10}+x^{9}+x^{8}+x^{7}+x^{5}+x^{4}+x^{2}+1$ \\
$g_{7}(x)$ & $x^{16}+x^{15}+x^{13}+x^{11}+x^{10}+x^{9}+x^{8}+x^{6}+x^{5}+x^{2}+1$ \\
$g_{8}(x)$ & $x^{16}+x^{14}+x^{13}+x^{12}+x^{9}+x_{8}+x^{6}+x^{5}+x^{2}+x+1$ \\
$g_{9}(x)$ & $x^{16}+x^{11}+x^{10}+x^{9}+x^{7}+x^{5}+1$ \\
$g_{10}(x)$ & $x^{16}+x^{14}+x^{13}+x^{12}+x^{10}+x^{8}+x^{7}+x^{5}+x^{2}+x+1$ \\
$g_{11}(x)$ & $x^{16}+x^{13}+x^{12}+x^{11}+x^{9}+x^{5}+x^{3}+x^{2}+x+1$ \\
$g_{12}(x)$ & $x^{16}+x^{12}+x^{11}+x^{9}+x^{7}+x^{6}+x^{5}+x+1$ \\
\hline
\end{tabular}




\section{Bit Interleavers}

After coding the message, in the ISDB-T $\mathrm{T}_{\mathrm{B}}$ system, there is an energy dispersal block, used to reduce interference between symbols that were generated by repetitive transmission. Connected to the output of the energy dispersal is the byte interleaver.

The byte interleaver is a convolutional interleaver where each input has a delay element (shift register) to delay the bits and group them in a different order in the output. This interleaver has twelve branches and seventeen delay elements as shown in Fig. 1 [6], [12].

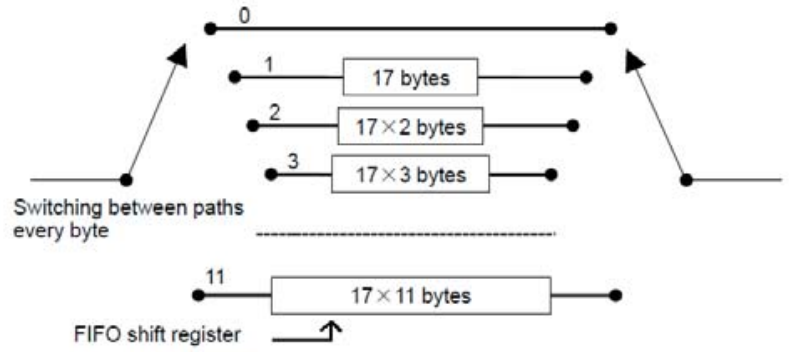

Fig. 1. ISDB- $\mathrm{T}_{\mathrm{B}}$ byte interleaver. From [12].

The bit interleaver for ISDB- $\mathrm{T}_{\mathrm{B}}$ is located after the convolutional encoder. It has a serial to parallel converter, so the input bits are divided into branches. The number of branches and the number of bits per branch depends on the modulation order, for example, a QPSK modulation requires two bits per branch. These groups of bits, called "symbols" at this point of the system, will later be mapped into the constellation points [6], [12].

ATSC 3.0 has only one-bit interleaver that is divided into three parts: Parity interleaver, Group-wise interleaver and Block interleaver.

The parity interleaver role in the system is to convert the staircase structure of the LDPC parity-check matrix into a quasi-cyclic structure that is similar to the information-part of the matrix [4], [14-15].

The Group-wise interleaver divides the code-word into groups of 360 bits and interleave these groups in an order defined in Annex B of [4].

Finally, the Block interleaver, which has two types, A and $\mathrm{B}$, will interleave the groups - the type of block interleaver depends on the LDPC code rate. All the algorithms of the ATSC 3.0 bit interleaver are described in sections 6.2 of [4].

\section{Constellations Mapper}

For the ISDB- $\mathrm{T}_{\mathrm{B}}$ system, there are four types of constellations: DQPSK, QPSK, 16QAM, and 64QAM. All four follow a table with the values of the constellation points, which are independent of the code rate used for the message. Fig. 2 illustrates the 64QAM constellation in the ISDB-T system [6], [12].

The constellations used in the ATSC 3.0 system are the NUC, due to the shapes of the constellations. Like in the
ISDB- $T_{B}$ system, these constellations follow a table with the values of the points, but the points vary according to the LDPC code rate [4], [14-15]. The available types are: QPSK, 16NUC, 64NUC, 256NUC, 1024NUC, and 4096NUC.

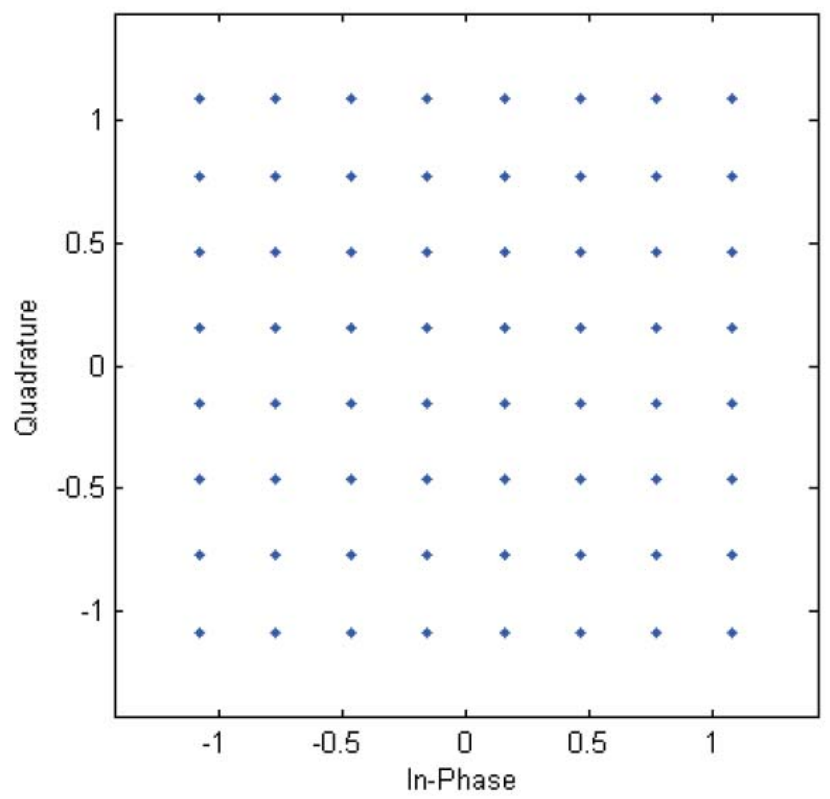

Fig. 2. ISDB- $\mathrm{T}_{\mathrm{B}}$ 64QAM Constellation. Adapted from [6].

Each constellation is optimized for the LDPC code rate, and the shapes generate a gain at the reception.

[16] shows that the $16 \mathrm{NUC}$ has a gain of $0,2 \mathrm{~dB}$ over the 16QAM when compared to the same signal-to-noise ratio. This gain increases along with the modulation order, for example, the 256NUC that has $1 \mathrm{~dB}$ gain over the 256QAM.

Fig. 3 illustrates the shape of a 64 NUC for LDPC code rate of $10 / 15$.

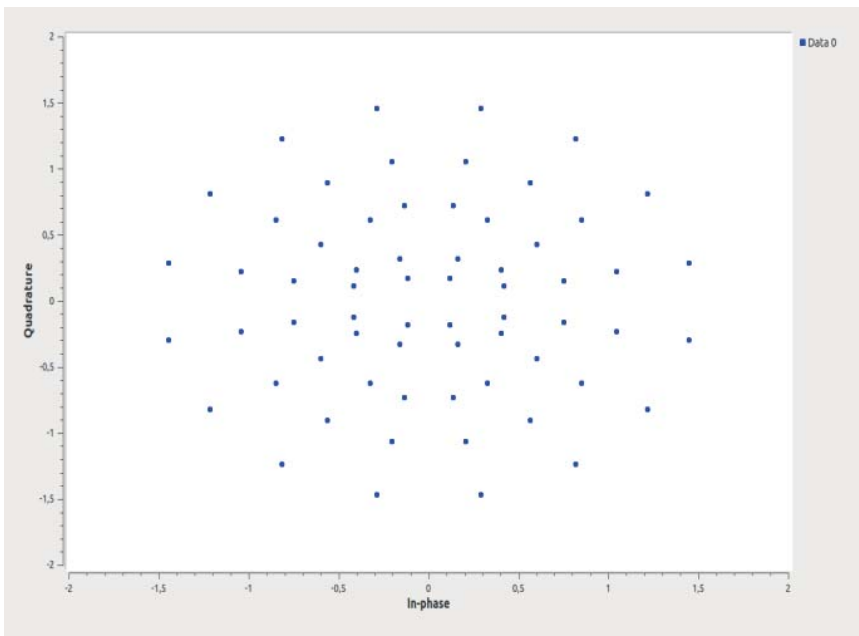

Fig. 3. ATSC 3.0 64NUC for 10/15 LDPC code rate. From [15]. 


\section{E. Spectrum Adjustment and LDM}

As ISDB- $\mathrm{T}_{\mathrm{B}}$ works with band segmentation, the spectrum adjustment stage is required to place de segments in order, as specified by [12]. Fig. 4 illustrates the order of the segments.

The segments can be arranged in up to three layers: A, B, and $\mathrm{C}$. Each layer is configured independently from another. The most common configuration used in Brazil is layer A to one segment (mobile) and layer B with twelve segments to HDTV [12], [17].

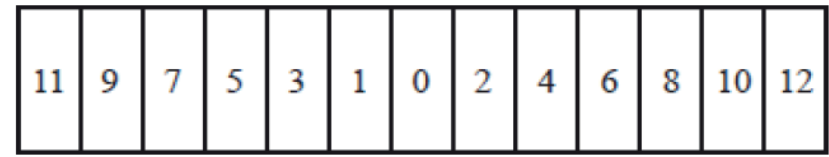

Fig. 4. ISDB- $\mathrm{T}_{\mathrm{B}}$ Segment Positions. Adapted from [6].

In ATSC 3.0, the LDM is used to multiplex the PLPs for transmission. Usually, there is the Core Layer (CL) PLP, which has a more robust configuration and the Enhanced Layer has a higher capacity setting.

The method used by the LDM to multiplex the two PLPs is attenuating the EL relatively to the CL using a factor alpha. After the attenuation, both layers are summed, and after the sum, a beta factor is applied to the resulting signal, so the overall power is normalized to $1 \mathrm{~W}$. The combination of both factors is called Injection Level (IL). That way both signals occupy the channel at the same time with a difference in power [4].

Fig. 5 illustrates the combination of the signals with the difference in power.

The ATSC 3.0 system also has the Frequency Division Multiplexing (FDM) and Time Frequency Division Multiplexing (TFDM) methods for multiples PLPs [4].

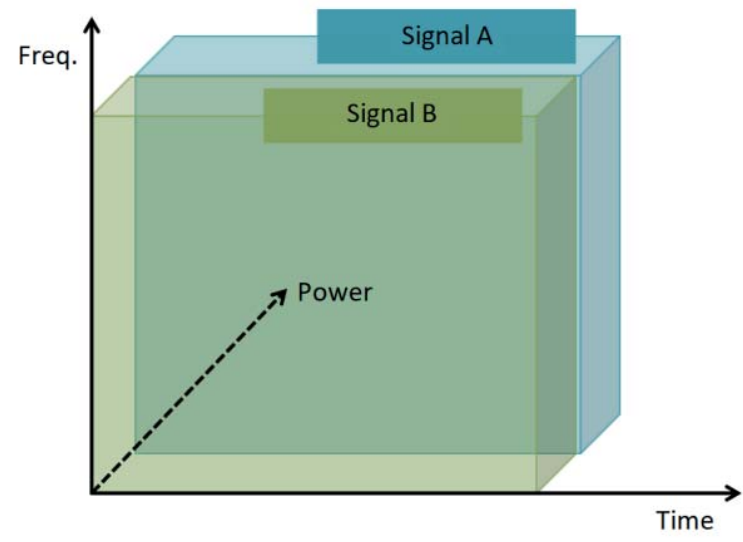

Fig. 5. Combined Signals with LDM. Adapted from [18]

\section{F. Time and Frequency Interleavers}

Both time and frequency interleaver stages are used to increase the error correction efficiency. Time interleaver increases robustness against impulsive noise and frequency interleaver against selective frequency fading [6].
In ISDB- $\mathrm{T}_{\mathrm{B}}$, the time interleaver is a convolutional interleaver (CI) that operates in all band segments separately, and they are cyclically combined in the output.

The frequency interleaver can be considered a block interleaver where the symbols are written in a memory and read in a certain order. It is divided into three parts: carrier interleaving between segments, intra-segment carrier rotation and carrier randomization. The three interleaving elements are detailed in [12].

The time interleaver in the ATSC 3.0 system has two types: CI and the hybrid time interleaver (HTI). The CI is similar to the time interleaver used in ISDB- $\mathrm{T}_{\mathrm{B}}$. More details about the CI and HTI can be found in section 7.1 of [4].

Frequency interleaving in the ATSC 3.0 system is made in all carriers of the OFDM symbol. Like the ISDB-T $\mathrm{T}_{\mathrm{B}}$, the symbols are written in the memory. On the output, for each IFFT size, there is a circuit that generates the interleaving order that the carriers are read. The address generating circuits are shown in section 7.3 of [4].

\section{G. Frame Structure}

The signal to be transmitted is organized in frames in both systems.

The OFDM symbols of the ISDB- $\mathrm{T}_{B}$ frames have three fixed sizes according to the IFFT used: Mode $1 \mathrm{~K}=1405$ (2k), Mode $2 \mathrm{~K}=2809$ (4k) and Mode $3 \mathrm{~K}=5617$ (8k) carriers in ISDB-T $\mathrm{T}_{\mathrm{B}}$. One frame consists in 204 OFDM symbols with 13 segments. The Scattered pilots (SP), continual pilots (CP), TMCC and Auxiliary Channel (AC) are inserted in the frame for transmission. Fig. 6 illustrates the frame structure for a coherent modulation [6], [12].

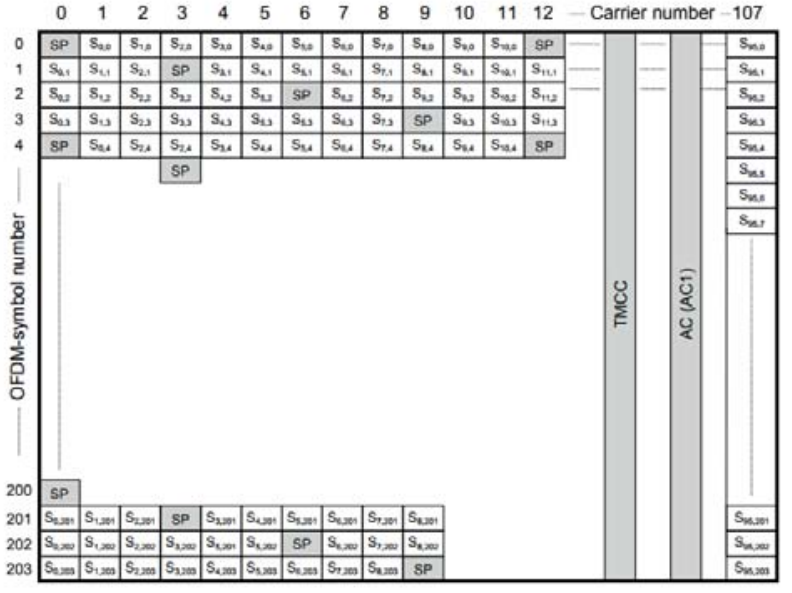

Fig. 6. ISDB- $\mathrm{T}_{\mathrm{B}}$ frame structure for a coherent modulation. From [12].

In ATSC 3.0, there are three IFFT sizes: 8k, 16k, and 32k. For each size, there are several useful carriers, 6913 for $8 \mathrm{k}$, 13825 for $16 \mathrm{k}$ and 27649 for $32 \mathrm{k}$. These numbers are variable according to a carrier reduction factor [4].

The signaling and control information is inserted in the first symbols of the frame, called preamble symbols. The 
useful data is included in the subframes. The pilots have inserted all symbols. Fig. 7 illustrates the frame structure for the ATSC 3.0 system [4], [14-15].

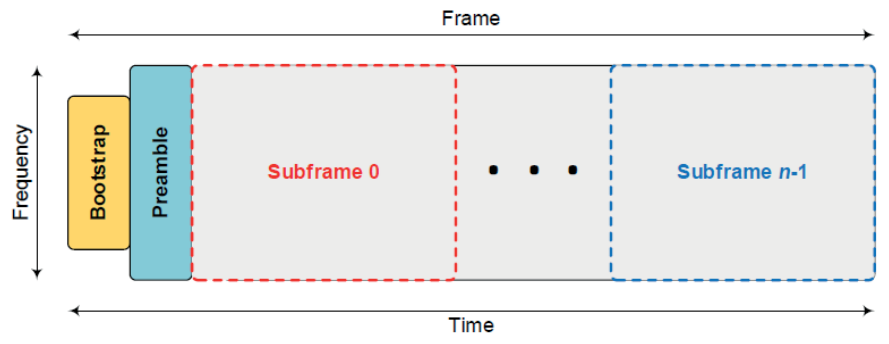

Fig. 7. ATSC 3.0 frame structure. From [4].

The first part of the frame is a signal called Bootstrap. This signal is added at the beginning of every frame, and it has different characteristics from the frame, for example, a smaller bandwidth, 4.5 $\mathrm{MHz}$, and smaller sampling rate, $6.144 \mathrm{Ms} / \mathrm{s}$ for a $6 \mathrm{MHz}$ channel and shorter IFFT, 2k.

The bootstrap signal is responsible for the synchronism; signal recovery; frequency and channel offset estimation and signaling information necessary to decode the rest of the frame [19].

\section{H. MISO and Peak-to-Average Power Ratio}

Since ATSC 3.0 supports not only SISO applications, it has a Transmit Diversity Code Filter Set that is a MISO predistortion technique that artificially decorrelates signals from multiple transmitters in SFN, minimizing the potential destructive interference.

There are two Peak-to-Average Power Ratio (PAPR) reduction technologies in the system: the Tone Reservation (TR) and the Active Constellation Extension (ACE). The TR reduces the PAPR by inserting specific cells in the OFDM symbols. The ACE reduces the PAPR by modifying the transmitted constellation points [4].

\section{Guard Interval}

The ISDB- $\mathrm{T}_{\mathrm{B}}$ and ATSC 3.0 systems utilize the guard interval after the IFFT. There are four options in the ISDB$\mathrm{T}_{\mathrm{B}}: 1 / 4,1 / 8,1 / 16$, and $1 / 32$.

In the ATSC 3.0 systems, the guard interval is expressed in samples of the OFDM symbol. There are twelve options: 192, 384, 512, 768, 1024, 1536, 2048, 2432, 3072, 3648, 4096, and 4864 samples.

The system also supports extra guard interval samples to make the total actual frame length equal to the signaled frame length. This configuration is used if the frame is time-aligned.

\section{BIT RATE AND CNR COMPARISONS}

The ATSC 3.0 system utilizes video compression standard H.265. The available literature and commercial disclosures suggest that bit rate requirements associated with various image qualities are similar to the ones listed in Table VI [20].

TABLE VI

SERVICE BitRATES. ADAPTED FROM [20]

\begin{tabular}{|c|c|}
\hline Service & Bitrate (Mbps) \\
\hline \hline Standard Definition (SD) & $0.8-1.8$ \\
High Definition (HD) \\
$\begin{array}{c}\text { Ultra High Definition } \\
\text { (UHD) }\end{array}$ & $2.0-3.5$ \\
\hline
\end{tabular}

The UHD service bitrate depends on the use of complementary techniques such as High Frame Rate (HFR), High Dynamic Range (HDR) and Wide Colour Gamut (WCG), therefore requires further specification [20].

Table $\mathrm{V}$ lists the system parameters and CNR requirements of both systems from tests in [21] and [22].

TABLE V

SYSTEM PARAMETERS AND CNR REQUIREMENTS. ADAPTED FROM [21] AND

\begin{tabular}{|c|c|c|c|}
\hline \multirow{2}{*}{ Configuration } & ISDB- $\mathrm{T}_{\mathrm{B}}$ & \multicolumn{2}{|c|}{ ATSC 3.0 } \\
\cline { 2 - 4 } & HD & $\begin{array}{c}\text { Mobile } \\
\text { HD }\end{array}$ & $\begin{array}{c}\text { Fixed } \\
\text { UHD }\end{array}$ \\
\hline \hline Modulation & $64-$ QAM & QPSK & $\begin{array}{c}64- \\
\text { QNUC } \\
\text { FEC }\end{array}$ \\
$3 / 4$ & $4 / 15$ & $10 / 15$ \\
Segments & 13 & - & - \\
IFFT & $8 \mathrm{k}$ & $16 \mathrm{k}$ & $16 \mathrm{k}$ \\
LDM Injection Level & - & 0 & -4 \\
(dB) & 20.1 & -0.2 & 18.7 \\
Required CNR (dB) & 19.32 & 2.5 & 21.4 \\
$\mathrm{R}_{\mathrm{b}}(\mathrm{Mbps})$ & & & \\
\hline
\end{tabular}

The tests show that with similar CNR it is possible to transmit UHDTV using ATSC 3.0 due to technologies applied in the system, while in ISDB- $\mathrm{T}_{\mathrm{B}}$ using all the segments it is possible to carry an HDTV signal. HDTV can be broadcasted in the CL of ATSC 3.0 with low CNR and bitrate.

Another important information is that configuring the ISDB- $_{B}$ system layer $A$ with one segment for Low Definition TV (LDTV) and layer B with twelve segments for HDTV, the CNR does not change for layer B. In the ATSC 3.0 system, the CNR would decrease without LDM transmission.

\section{CONCLUSION}

This review presented a comparison between the physical layer of the ISDB- $\mathrm{T}_{\mathrm{B}}$ and ATSC 3.0 systems. 
The technologies implemented in ATSC 3.0, for example, 32k IFFT, LDPC and BCH codes, NUC, LDM and H.265 video compression enable robustness and high transmission rates, which are proper for the transmission of UHDTV signals and satisfies the current scenario of Communications systems.

The ISDB- $\mathrm{T}_{\mathrm{B}}$ system presents fewer settings options when compared to the ATSC 3.0. Therefore, the present work serves as a guide to the development of a new broadcast system for Brazil.

\section{ACKNOWLEDGMENTS}

The authors would like to thank PPGEEC and their colleagues at Mackenzie's Digital TV Research Laboratory.

\section{REFERENCES}

[1] Y. Wu, B. Rong, K. Salehian and G. Gagnon, "Cloud Transmission: A New Spectrum-Reuse Friendly Digital Terrestrial Broadcasting Transmission System," in IEEE Transactions on Broadcasting, vol. 58, no. 3, pp. 329-337, September 2012.

[2] Y. Wu et al., "Comparison of Terrestrial DTV Transmission Systems: The ATSC 8-VSB, the DVB-T COFDM, and the ISDB-T BST-OFDM," in IEEE Transactions on Broadcasting, vol 46, no. 2, pp.101-113, June 2000.

[3] L. Fay, L. Michael, D. Gómez-Barquero, N. Ammar and M. W. Caldwell, "An Overview of the ATSC 3.0 Physical Layer Specification," in IEEE Transactions on Broadcasting, vol. 62, no. 1, pp. 159-171, March 2016.

[4] Advanced Television Systems Committee. "ATSC Standard: Physical Layer Protocol (A/322). Doc. A/322:2106." Washington, D.C, September 2016.

[5] Digital Video Broadcasting. "Frame Structure channel coding and modulation for a second generation digital terrestrial television broadcasting system (DVB-T2)." France, 2011.

[6] C. Akamine and Y. Iano, "Simulation Software for the ISDBTв Modulation System," in SET International Journal of Broadcast Engineering - SET IJBE v. 1, Article 3, 11p, 2015.

[7] Advanced Television Systems Committee. "ATSC Standard: Link-Layer Protocol (A/330). Doc. A/330:2016." Washington, D.C, September 2016.

[8] Advanced Television Systems Committee. "ATSC Standard: Audio Common Elements (A/342-1). Doc. A/342-1:2017." Washington, D.C, January 2017.

[9] Advanced Television Systems Committee. "ATSC Standard: AC-4 System (A/342-2). Doc. A/342-2:2017." Washington, D.C, February 2017.

[10] Advanced Television Systems Committee. "ATSC Standard: MPEG-H System (A/342-3). Doc. A/342-3:2017." Washington, D.C, March 2017.

[11] Advanced Television Systems Committee. "ATSC Standard: Video - HEVC (A/341). Doc. A/341:2017." Washington, D.C, May 2017.

[12] Associação Brasileira de Normas Técnicas. "Televisão Digital terrestre - Sistema de Transmissão," ABNT NBR 15601:2007, 2007.
[13] I. E. Bocharova, B. D. Kudryashov and R. Johannesson, "LDPC convolutional codes versus QC LDPC block codes in communication standard scenarios," 2014 IEEE International Symposium on Information Theory, Honolulu, HI, 2014, pp. 2774-2778.

[14] V. Dionísio, C. Akamine, "Desmistificando o padrão de TV digital ATSC 3.0," 2016 Brazilian Technology Symposium, Campinas, $6 \mathrm{p}$.

[15] V. Dionísio, C. Akamine, "ATSC 3.0 implementation in GNURadio Companion,” 2017 IEEE International Symposium on Broadband Multimedia Systems and Broadcasting, Cagliari, 2017, 6p.

[16] N. S. Loghin, J. Zöllner, B. Mouhouche, D. Ansorregui, J. Kim and S. I. Park, "Non-Uniform Constellations for ATSC 3.0," in IEEE Transactions on Broadcasting, vol. 62, no. 1, pp. 197203, March 2016.

[17] G. H. M. G. de Oliveira, C. Akamine, and Y. P. Maciel, "Implementation of ISDB-T LDM broadcast system using LDPC codes," 2016 IEEE International Symposium on Broadband Multimedia Systems and Broadcasting (BMSB), Nara, 2016, pp. 1-4.

[18] S. I. Park et al., "Low Complexity Layered Division Multiplexing for ATSC 3.0," in IEEE Transactions on Broadcasting, vol. 62, no. 1, pp. 233-243, March 2016.

[19] Advanced Television Systems Committee. "ATSC Standard: System Discovery and Signaling (A/321). Doc. A/321:2106." Washington, D.C, March 2016.

[20] C. Regueiro et al., "Network planning for local service in ATSC 3.0 single frequency networks," 2017 IEEE International Symposium on Broadband Multimedia Systems and Broadcasting (BMSB), Cagliari, Italy, 2017, pp. 1-5.

[21] P. Guedes Esperante, C. Akamine, and G. Bedicks, "Comparison of Terrestrial DTV Systems: ISDB-TB and DVB-T2 in $6 \mathrm{MHz}$," in IEEE Latin America Transactions, vol. 14, no. 1, pp. 45-56, Jan. 2016.

[22] ETRI, "NAB 2017 ATSC 3.0 Presentation," Las Vegas, United States of America, 2017.

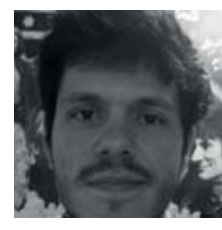

Victor Morales Dionísio received his B.Sc degree in Electrical Engineering and his M.Sc. degree in Electrical Engineering from Mackenzie Presbyterian University, São Paulo, Brazil, in 2010 and 2017, respectively. His research interests are in broadcast systems and Software Defined Radio.

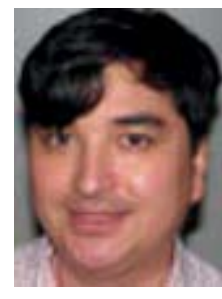

Cristiano Akamine received his B.Sc. degree in Electrical Engineering from Mackenzie Presbyterian University, São Paulo, Brazil, in 1999. He received his M.Sc. and Ph.D. degree in Electrical Engineering from the State University of Campinas (UNICAMP), São Paulo, Brazil, in 2004 and 2011 respectively. He is a professor of Embedded Systems, Software Defined Radio and

Advanced Communication Systems at Mackenzie Presbyterian University. $\mathrm{He}$ is a researcher in the Digital TV Research Laboratory at Mackenzie Presbyterian University since 1998, where he has had the opportunity to work with many digital TV systems. His research interests are in a system on chip for broadcast TV and Software Defined Radio 